\title{
The effectiveness of an endotracheal tube holder
}

Byung Gun $\operatorname{Lim}^{1 *}$

1. Department of Anesthesiology and Pain Medicine, Korea University Guro Hospital, Korea University College of Medicine, Seoul, South Korea

The security of the endotracheal tube (ETT) is important in the anesthesia and intensive care settings. Recently, an ETT holder instead of an adhesive tape is frequently used to provide the fixation of the ETT. There are some studies that focused on the effectiveness of the ETT holder in preventing displacement of ETT compared to an adhesive tape $(1,2)$. I have experienced the use of an ETT holder (E-holder, KS medical, Bucheon, Korea) in many different anesthesia settings and recognized its usefulness and convenience other than ETT security in the following specific settings.

Firstly, the ETT holder is definitely effective for patients undergoing procedures that frequently require the adjustment of ETT (or double lumen tube) location including thoracic surgery. The location of double lumen tube needed for lung separation can be modified for effective ventilation during anesthetic induction or surgery, and after position changes. The tube can be easily relocated to the proper place for lung separation and effective ventilation by unlocking/relocking the button of the ETT holder under flexible bronchoscopic guidance (Fig. 1A), which can facilitate the operative procedure to be performed easily and quickly.

Secondly, the ETT holder can be useful in procedures requiring the prone position (3). Especially, it is useful in all circumstances requiring the adjustment of ETT in the prone position. For instance, it is definitely useful for patients with Duchene muscular dystrophy undergoing scoliosis surgery under prone position. Adolescent patients with Duchene muscular dystrophy frequently have tracheobronchial malacia. If so, the airway pressure can be elevated due to the narrowing of the lesion of malacia when changed to the prone position (4). To overcome this problem, a reinforced ETT should be relocated so that it passes the narrowed tracheal lesion under flexible bronchoscopic guidance (Fig. 1B). However, the approach for this management is somewhat challenging with the patient in the prone position, and especially, the release and refixation of ETT with an adhesive tape is very difficult. The ETT can be easily relocated to the proper place to overcome the narrowing part of trachea by unlocking/relocking the button of the ETT holder, which can prevent the risk of respiratory complications associated with

\section{Corresponding author:}

Byung Gun Lim, MD, PhD

Department of Anesthesiology and Pain Medicine, Korea University College of Medicine, Guro Hospital, 148

Gurodong-ro, Guro-gu, Seoul, Korea

Tel: +82+2-2626-1437 Fax: +82+2-26261438 E-mail address: bglim9205@korea.ac.kr

Receive date: 2015-10-05 | Accept date: 2015-10-25| Publish date: 2015-12-01

DOI: 10.7575/aiac.abcmed.16.04.01.01 
ventilatory difficulty and secure the safety of patients.

Thirdly, the ETT holder is effective in keeping the ETT in a midline position. This is especially so when a flexible laryngeal mask airway (Flexible LMATM, Wooyoung meditech, Seoul, Korea; FLMA) is used because maintaining a midline position of LMA is very important in securing ventilation without leakage (Fig. 1C, 1D). Although an adhesive tape without a bite block or oral airway may enable an FLMA to be kept in a midline position, there is still a risk of airway obstruction associated with the patient biting an FLMA. Moreover, considering that an FLMA displays a tendency to be pushed out from the pharyngolaryngeal region if it is positioned so that only a low grade sealing is achieved, a tight fixation of the FLMA using the ETT holder is very important.

I suggest that the ETT holder can enhance the convenience of anesthesiologists while providing more useful anesthetic management to patients in the above anesthesia settings.
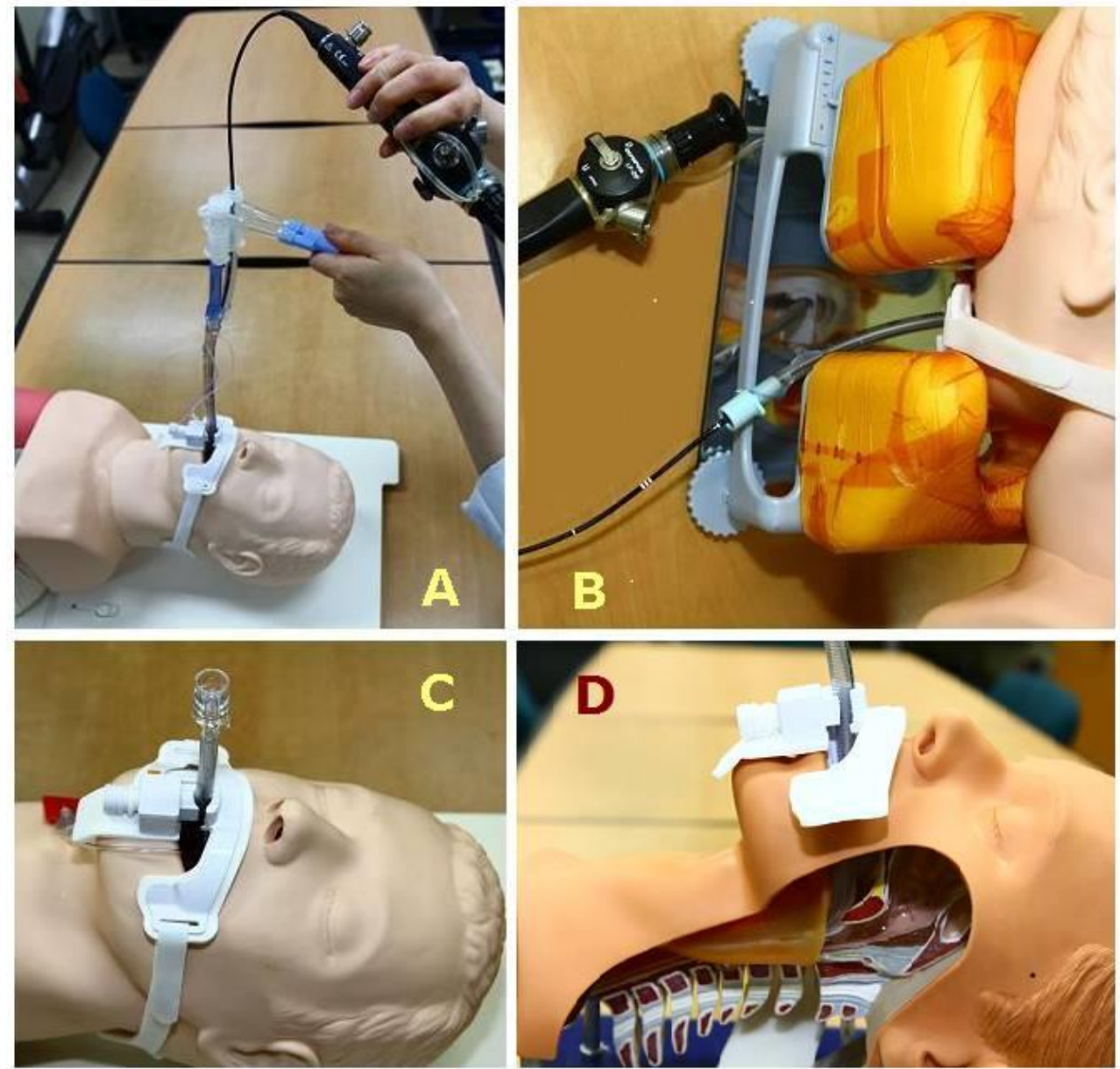

Figure 1: Use of an endotracheal tube holder in various anesthesia settings. (A) Repositioning of a double lumen tube with a flexible bronchoscope in thoracic surgery. (B) Repositioning of an endotracheal tube with a flexible bronchoscope in a surgery requiring the prone position. (C) Fixation of a flexible laryngeal mask airway (a front view). (D) Fixation of a flexible laryngeal mask airway (a lateral view). 
Conflicts of Interest: None

\section{References}

1. Murdoch $E$, Holdgate A. A comparison of tape-tying versus a tube-holding device for securing endotracheal tubes in adults. Anaesth Intensive Care 2007; 35:730-5.

2. Shimizu T, Mizutani T, Yamashita S, Hagiya K, Tanaka M. Endotracheal tube extubation force: adhesive tape versus endotracheal tube holder. Respir Care 2011; 56:1825-9.

3. Santhosh MC, Torgal SV, Pai RB, Roopa S, Santoshi VB, Rao RP. Comparison of tube-taping versus a tube-holding device for securing endotracheal tubes in adults undergoing surgery in prone position. Acta Anaesthesiol Belg 2013; 64:75-9.

4. Lee DK, Lim BG, Lee IO, Oh HR, Lim SH, Lee MK. Unexpected tracheal narrowing during general anesthesia in the prone position of Duchenne muscular dystrophy patient -A report of two cases-. Korean J Anesthesiol 2013; 64:456-9. 